\title{
Anatomical Variations of the Biliary Tree Found with Endoscopic Retrograde Cholagiopancreatography in a Referral Center in Southern Iran
}

\author{
Seyed Alireza Taghavi ${ }^{1}$, Ramin Niknam ${ }^{1, *}$, Seyed Ehsan Alavi ${ }^{1}$, Fardad Ejtehadi ${ }^{1}$, \\ Gholam Reza Sivandzadeh ${ }^{1}$, Ahad Eshraghian ${ }^{1}$
}

1. Gastroenterohepatology Research Center, Shiraz University of Medical Sciences, Shiraz, Iran

\section{* Corresponding Author:}

Ramin Niknam, MD

Gastroenterohepatology Research Center, Nemazee Hospital, PO Box: 71937-11351 Shiraz, Iran.

Telefax: +987136281442

Email: niknamramin@yahoo.com

Received: 09 Jun. 2017

Accepted: 18 Sep. 2017

\section{ABSTRACT}

\section{BACKGROUND}

Anatomical variations in the biliary system have been proven to be of clinical importance. Awareness of the pattern of these variations in a specific population may help to prevent and manage biliary injuries during surgical and endoscopic procedures. Knowledge of the biliary anatomy will be also of great help in planning the drainage of adequate percentage of liver parenchyma in endoscopic or radiological procedures.

\section{METHODS}

All consecutive patients undergoing endoscopic retrograde cholangiopancreatography (ERCP) from April 2013 to April 2015 at Nemazee Hospital, a referral center in the south of Iran, were included in this cross-sectional study. The patients with previous hepatic or biliary surgery, liver injury or destructive biliary disease were excluded from the study. All ERCPs were reviewed by two expert gastroenterologists in this field. The disagreed images by the two gastroenterologists were excluded. Huang classification was used for categorizing the different structural variants of the biliary tree, and the frequency of each variant was recorded.

\section{RESULTS}

Totally, 362 patients (181 men and 181 women) were included in the study. 163 patients (45\%) had type A1 Huang classification (right dominant), which was the most prevalent type among our patients. $55 \%$ of them had non-right dominant anatomy. The result of the Chi-square test revealed that there was no statistically significant difference between the men and women regarding the anatomical variations $(p=0.413)$.

\section{CONCLUSION}

The anatomical variation in the biliary system among Iranian patients is comparable to other regions of the world. Significant proportions of our patients are non-right dominant and may need bilateral biliary drainage.

\section{KEYWORDS:}

Endoscopic retrograde cholangiopancreatography, Biliary anatomy, Huang classification

Please cite this paper as:

Taghavi SA, Niknam R, Alavi SE, Ejtehadi F, Sivandzadeh GR, Eshraghian A. Anatomical Variations of the Biliary Tree Found with Endoscopic Retrograde Cholagiopancreatograhy in a Referral Center in Southern Iran. Middle East J Dig Dis 2017;9:201-205. doi: $10.15171 /$ mejdd.2017.74.

\section{INTRODUCTION}

Anatomical variations in the biliary system have been proven to be of clinical importance. Open surgical and endoscopic procedures such as liver resection, cholecystectomy, liver transplantation, and endoscopic or radiological drainage of obstructions are now increasingly performed on the biliary system and may have serious life threatening complications. ${ }^{1}$ Understanding the normal anatomy and anatomical variations in the biliary tree within a specific population is of great importance both in preventing and minimizing these complications, and 

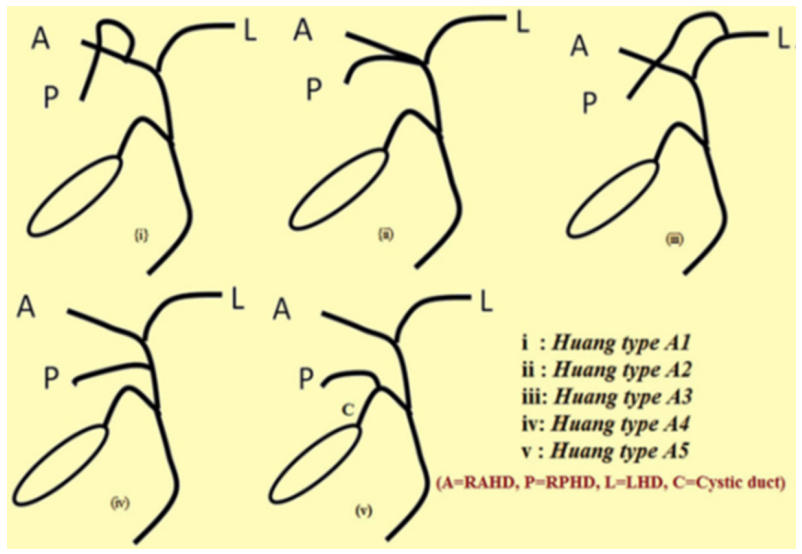

A = RAHD: Right anterior hepatic duct, $\mathrm{P}=$ RPHD: Right posterior hepatic duct, $\mathrm{L}=$ LHD: Left hepatic duct

Fig.1: Huang classification of the biliary tract anatomy.

ensuring that adequate and effective surgical, radiological, or endoscopic procedures have been performed., ${ }^{2,3}$

There are several imaging modalities for evaluation of the diseases of the biliary tree. Conventional and endoscopic ultrasonography (EUS), endoscopic retrograde cholangiopancreatography (ERCP), magnetic resonance cholangiopancreatography (MRCP), and computed tomography (CT) are now widely available and applied in equipped centers for this purpose. ${ }^{4-6}$ ERCP is not only the gold standard method for the diagnosis of the abnormalities of the biliary tree, but also has therapeutic benefits over other imaging modalities. ${ }^{7}$

Biliary drainage of the liver parenchyma has multiple normal anatomical variants. Several classification methods have been proposed for anatomical variations in the biliary tree. ${ }^{89}$ Huang classification has been proposed by Huang and co-workers in 1996 based on the right posterior hepatic bile duct insertion and is primarily used for donors in living donor liver transplantation. ${ }^{10}$ Five groups have been introduced in Huang classification, with A1 as the most common and A5 as the least common types.

This study aimed to investigate the frequencies of different anatomical variations in a cluster of Iranian population from a referral center in South of Iran referred for ERCP based on Huang classification.

\section{MATERIALS AND METHODS}

In a cross-sectional study, we included all consecutive patients undergoing ERCP from 2014 to April 2016 at Nemazee Hospital, Shiraz, Iran. Nemazee Hospital is a
Table 1: The frequencies of anatomical variants of the biliary system based on Huang classification

\begin{tabular}{lccc}
\hline \multicolumn{1}{c}{ Anatomical variant } & \multicolumn{2}{c}{ Sex } & Total \\
\hline & Women & Men & \\
\hline Huang type A1 (\%) & $83(22.9)$ & $80(22.1)$ & $163(45)$ \\
\hline Huang type A2 (\%) & $38(10.5)$ & $40(11)$ & $78(21.5)$ \\
\hline Huang type A3 (\%) & $29(8.1)$ & $19(5.2)$ & $48(13.3)$ \\
\hline Huang type A4 (\%) & $5(1.4)$ & $8(2.2)$ & $13(3.6)$ \\
\hline Huang type A5 (\%) & 0 & 0 & 0 \\
\hline Non Huang type $(\%)$ & $26(7.2)$ & $34(9.4)$ & $60(16.6)$ \\
\hline Total & 181 & 181 & 362 \\
\hline
\end{tabular}

referral center for ERCP in southern Iran and is affiliated to Shiraz University of Medical Sciences, Shiraz, Iran. The patients with previous hepatic or biliary surgery or injury or destructive biliary disease were excluded from the study. Huang classification was used for categorizing the different structural variants of the biliary tree, and the frequency of each variant was recorded. Five anatomical variants of the biliary tree are defined in Huang classification (figure 1).

Huang type A1 (right-dominant): the right posterior duct drains into the right anterior hepatic ducts and joins the left hepatic duct to form the common hepatic duct (CHD). Huang type A2 (trifurcation): the right posterior hepatic duct, right anterior hepatic ducts and left hepatic duct join each other in a trifurcation and form CHD. Huang type A3 (left dominant): the right posterior hepatic duct drains into the left hepatic duct and then joins the right anterior hepatic duct to from CHD. Huang type A4 (aberrant right): the right anterior hepatic duct drains into the left hepatic duct and then joins the right posterior hepatic duct to from CHD. Huang type A5 (aberrant right): the right anterior hepatic duct drains into the left hepatic duct and forms CHD. The right posterior hepatic duct drains into the cystic duct. Anatomical variants that were not compatible to Huang classification were defined as non-Huang type. All procedures were done by two gastroenterologists. All cholangiopancreatograms were reviewed by the same two expert gastroenterologists in this field. The disagreed images by the two gastroenterologists were excluded from the study.

The study protocol was approved by the Ethics Committee of Shiraz University of Medical Sciences. The study was explained for the participants and written 


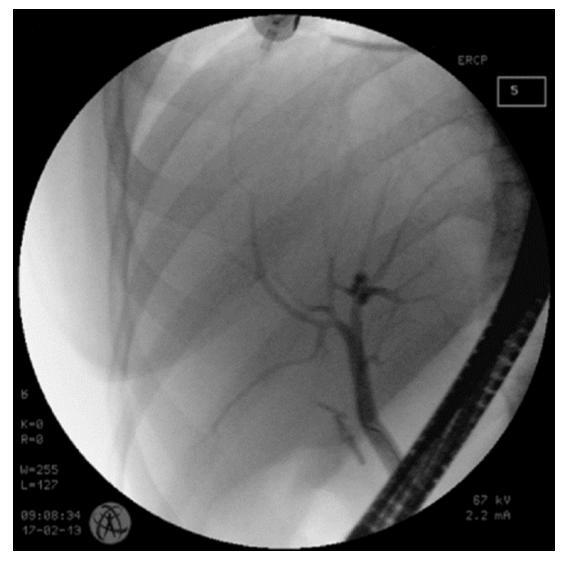

Fig.2: Huang type A1

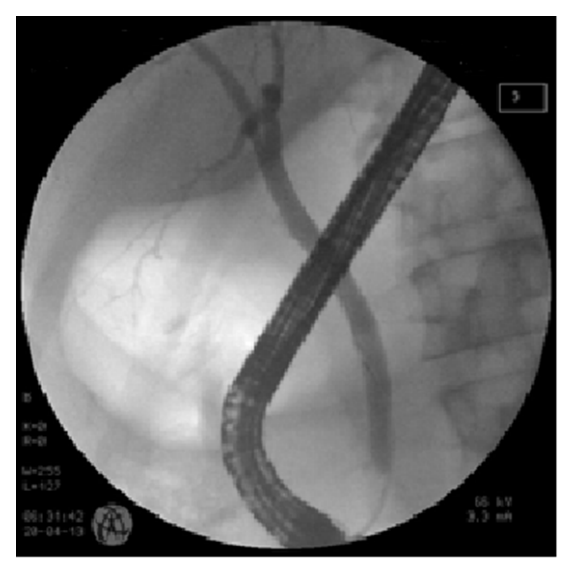

Fig.4: Huang type A3

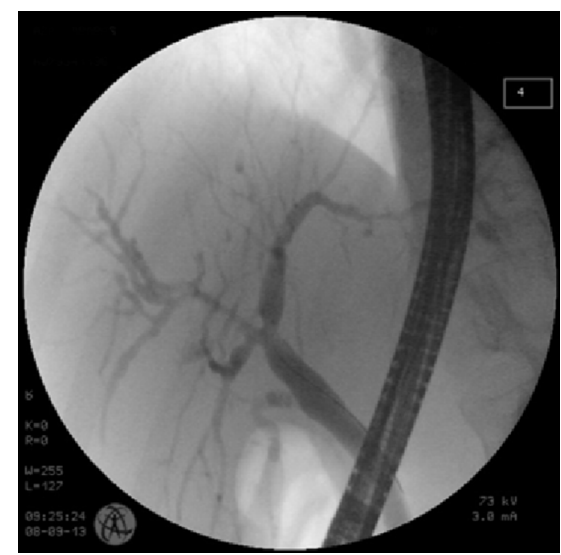

Fig.3: Huang type A2

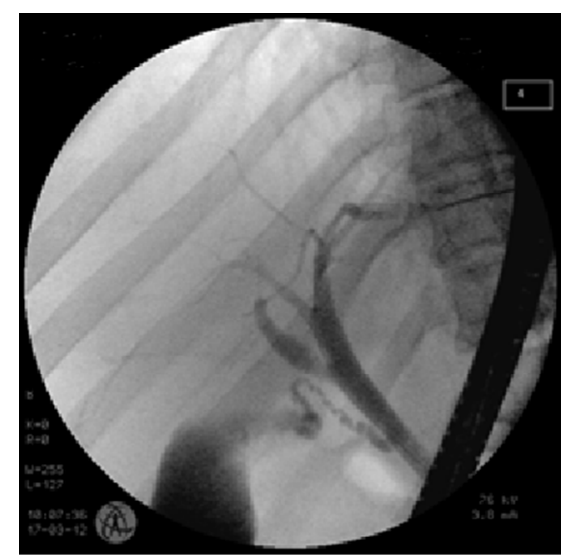

Fig.5: Huang type A4 informed consents were obtained.

The optimal sample size was calculated to be 320 patients according to Morgan table. Comparison of continuous variables was performed with the Student $t$ test, and categorical variables were compared using the Chisquare test. Data were presented using means \pm standard deviation for numerical variables, and percentages and counts for categorical variables. Statistical analysis was performed using SPSS software version 16.0 (SPSS Inc.; Chicago, IL, USA). $p$ values $<0.05$ was considered as statistically significant.

\section{RESULTS}

Of the 413 patients who underwent ERCP during the study period, a total of 362 patients (181 men and 181 women) were included in the study. 51 patients were excluded due to unsatisfactory or disagreed images. 163 patients had type A1 Huang (right dominant) clas- sification. The prevalence of A1 Huang (right dominant) classification was $45 \%$ that was the most prevalent type among our patients. Type A2 Huang (trifurcation) was seen in 78 patients (21.5\%). Type A3 Huang (left dominant) was seen in 48 patients (13.3\%). Type A4 was seen in 13 patients (3.6\%). There was no patient categorized as type 5. Non-right dominant anatomy was seen in 55\% of our patients. The frequencies of anatomical variants are shown in table 1.

Using Chi-square test, there was no statistically significant difference between men and women regarding the anatomical variation $(p=0.413)$. Figures $2-5$ show anatomical variants classified as Huang type A1-A4.

\section{DISCUSSION}

Our study determined the frequencies of each anatomical variation in the biliary system based on Huang classification in a cluster of Iranian population. Type A 
was the most prevalent one among our study population. Other studies in other regions of the world have reported variation in the biliary tree based on Huang classification. In a descriptive study, MRCP was used to evaluate the anatomical alterations in the biliary tree among a selected Egyptian population. Similar to our results, in the mentioned study type A1 classification was the most prevalent one, which was seen in over $60 \%$ of Egyptian population. This is much higher compared with our results. ${ }^{11}$ Huang and colleagues reported the prevalence of type A classification to be $63 \%$ in Chinese population using ERCP method. ${ }^{10}$ Cheng and co-workers reported similar results in Chinese population. ${ }^{12}$ Moreover, Kitami and others reported the type and variations of the biliary tract using multi-detector CT cholangiography. ${ }^{13}$ The reported percentages of Huang type A1, A2, A3, A4 and A5 in their study were $73 \%, 5 \%, 12 \%, 4 \%$, and $3 \%$, respectively. In North American population, Kapoor and colleagues compared MRCP and intraoperative cholangiography to determine the anatomical variations in the biliary tree and concluded that MRCP was comparable to intraoperative cholangiography for showing the anatomy of the biliary tree. The reported frequencies of type A1, $\mathrm{A} 2, \mathrm{~A} 3, \mathrm{~A} 4$, and $\mathrm{A} 5$ in their study were $63 \%, 0 \%, 8 \%$, $8 \%$, and $0 \%$, respectively. ${ }^{14}$ Another study on American population using MRCP to evaluate anatomical variations in adult-to-adult living liver transplantation showed $73 \%$ prevalence of type A1 in the studied population. ${ }^{15}$ Two other studies on Turkish population showed the frequency of type A1 to be $8-29 \%$, which is significantly lower than that in Chinese, American, and Iranian population. ${ }^{16-17}$ In a study in Germany, the authors compared MRCP and ERCP for evaluation of living donor anatomy and concluded that ERCP was safe and superior to MRCP for detection of biliary variations before liver transplantation. ${ }^{18}$ In another Asian study, type A1 Huang classification was reported to be observed in $65 \%$ of the study population. ${ }^{19}$ In Korean series of 300 living liver donors, Choei and co-workers showed type A1 (conventional type) in $63 \%$ of the population with intraoperative cholangiography.20 10\% of them had trifurcation (type A2) and 11\% had type C. ${ }^{20}$

The second most prevalent type was A3 in most studies $^{9,14,15,21}$, except for Chinese population. Type A3 may be accompanied by more injuries in the living donor liver transplantation and may need double anastomosis to avoid biliary leakage after surgery. ${ }^{9}$ However, in our study, type A2 Huang classification was the second most prevalent type. The drainage of the right posterior hepatic duct into the CHD (type A4) and cystic duct (type A5) may cause the surgeons to resect or ligate the aberrant duct, leading to further injuries and biliary leakage after surgery. ${ }^{22}$ Type A4 was seen in a small proportion of our study population and none of our patients was categorized as having type A5.

The above-mentioned findings have clinical implications in ERCP as well as biliary or hepatic surgery. High prevalence of type A means that in most of the cases of hilar biliary obstruction, drainage of the main stem right biliary duct will ensure the drainage of the majority of the liver parenchyma and will be the minimal adequate procedure in palliation of obstructive symptoms. In contrast, isolated drainage of the left system is likely to have suboptimal results. Differentiation of the second most common variant (Huang B) will be of outmost importance since in this variant no "main" right hepatic duct is present and the minimal adequate palliative procedure will require drainage of two out of three (right anterior, right posterior, and left) ducts. In this situation, placing a single stent in the right system will most likely provide an inadequate drainage

\section{CONCLUSION}

This study is the first study showing the anatomical variations of the biliary system in Iranian population. In conclusion, the anatomical variation in the biliary system of the Iranian patients is comparable to other regions of the world.

\section{ACKNOWLEDGEMENTS}

This study was extracted from Ehsan Alavi's thesis submitted to the School of Medicine as a partial fulfillment of the requirements for degree of specialty in internal medicine. The authors would like to thank Shiraz University of Medical Sciences, Shiraz, Iran and also the Center for Development of Clinical Research of Nemazee Hospital and Dr. Nasrin Shokrpour for editorial assistance.

\section{CONFLICT OF INTEREST}

The authors declare no conflict of interest related to this work. 


\section{REFERENCES}

1. Crawford JM. Development of the intrahepatic biliary tree. Semin Liver Dis 2002;22:213-26. doi: 10.1055/s-2002-34508.

2. Desmet VJ. Pathogenesis of ductal plate malformation. $J$ Gastroen Hepatol 2004;19:S356-60.

3. Gazelle GS, Lee MJ, Mueller PR. Cholangiographic segmental anatomy of the liver. RadioGraphics 1994;14:100513. doi: 10.1148/radiographics.14.5.7991810.

4. Melamud K, LeBedis CA, Anderson SW, Soto JA. Biliary imaging: multimodality approach to imaging of biliary injuries and their complications. Radiographics 2014;34:613-23. doi: 10.1148/rg.343130011.

5. Hyodo T, Kumano S, Kushihata F, Okada M, Hirata M, Tsuda T, et al. CT and MR cholangiography: advantages and pitfalls in perioperative evaluation of biliary tree. $\mathrm{Br}$ J Radiol 2012;85:887-96. doi: 10.1259/bjr/21209407.

6. Saad WE, Ginat D. Computed tomography and magnetic resonance cholangiography. Tech Vasc Interv Radiol 2008;11:74-89. doi: 10.1053/j.tvir.2008.07.002.

7. ASGE Training Committee, Jorgensen J, Kubiliun N, Law JK, Al-Haddad MA, Bingener-Casey J, et al. Endoscopic retrograde cholangiopancreatography (ERCP): core curriculum. Gastrointest Endosc 2016;83:279-89. doi: 10.1016/j.gie.2015.11.006.

8. Champetier J. Les voies biliaires. In: Chevrel JP (ed) Anatomie clinique, Le tronc. 1994; Springer, Paris, p 41.

9. Ohkubo M, Nagino M, Kamiya J, Yuasa N, Oda K, Arai T, et al. Surgical anatomy of the bile ducts at the hepatic hilum as applied to living donor liver transplantation. Ann Surg 2004;239:82-6. doi:10.1097/01. sla.0000102934.93029.89.

10. Huang TL, Cheng YF, Chen CL, Lee TY. Variants of the bile ducts: clinical application in the potential donor of living-related hepatic transplantation. Transplant Proc 1996;28:1669-70.

11. Tawab MA, Taha Ali TF. Anatomic variations of intrahepatic bile ducts in the general adult Egyptian population: 3.0-T MR cholangiography and clinical importance. Egypt J Radiol Nuclear Med 2012;43:111-7. doi:10.1016/j.ejrnm.2012.02.004.

12. Cheng YF, Huang TL, Chen CL, Chen YS, Lee TY. Variations of the intrahepatic bile ducts: application in living related liver transplantation and splitting liver transplantation. Clin Transplant 1997;11:337-40.

13. Kitami M, Takase K, Murakami G, Ko S, Tsuboi M, Saito $\mathrm{H}$, et al. Types and frequencies of biliary tract variations associated with a major portal venous anomaly: analysis with multi-detector row CT cholangiography. Radiology 2006;238:156-66. doi: -10.1148/radiol.2381041783.

14. Kapoor V, Peterson MS, Baron RL, Patel S, Eghtesad B, Fung JJ. Intrahepatic biliary anatomy of living adult liver donors: correlation of mangafodipir trisodium-enhanced MR cholangiography and intraoperative cholangiography. AJR Am J Roentgenol 2002;179:1281-6. doi: 10.2214/ajr.179.5.1791281.
15. Limanond P, Raman SS, Ghobrial RM, Busuttil RW, Lu DS. The utility of MRCP in preoperative mapping of biliary anatomy in adult-to-adult living related liver transplant donors. J Magn Reson Imaging 2004;19:209-15. doi:10.1002/jmri.10446.

16. Karakas HM, Celik T, Alicioglu B. Bile duct anatomy of the Anatolian Caucasian population: Huang classification revisited. Surg Radiol Anat 2008;30:539-45. doi:10.1007/ s00276-008-0365-y.

17. Düşünceli E, Erden A, Erden I. Anatomic variations of the bile ducts: MRCP findings. Tani Girisim Radyol 2004;10:296-303. Turkish.

18. Wietzke-Braun P, Braun F, Muller D, Lorf T, Ringe B, Ramadori G. Adult-to adult right lobe living donor liver transplantation: comparison of endoscopic retrograde cholangiography with standard T2-weighted magnetic resonance cholangiography for the evaluation of donor biliary anatomy. World J Gastroenterol 2006;12:5820-5. doi: 10.3748/wjg.v12.i36.5820.

19. Thungsuppawattanakit P, Arjhansiri K. Anatomic variants of intrahepatic bile ducts in Thais. Asian Biomed 2012;6:51-7.

20. Choi JW, Kim TK, Kim KW, Kim AY, Kim PN, Ha HK, et al. Anatomic variation in intrahepatic bile ducts: an analysis of intraoperative cholangiograms in 300 consecutive donors for living donor liver transplantation. Korean J Radiol 2003;4:85-90. doi: 10.3348/kjr.2003.4.2.85.

21. Kim RD, Sakamoto S, Haider MA, Molinari M, Gallinger S, McGilvray ID, et al. Role of magnetic resonance cholangiography in assessing biliary anatomy in right lobe living donors. Transplantation 2005;79:1417-21.

22. Mortelé KJ, Ros PR. Anatomic variants of the biliary tree: MR cholangiographic findings and clinical applications. AJR Am J Roentgenol 2001;177:389-94. doi: 10.2214/ ajr.177.2.1770389. 